\title{
Spectroscopy of the growing circumstellar disk in the $\delta$ Scorpii Be binary ${ }^{\star}$
}

\author{
A. S. Miroshnichenko ${ }^{1,2}$, K. S. Bjorkman ${ }^{1}$, N. D. Morrison ${ }^{1}$, J. P. Wisniewski ${ }^{1}$, N. Manset ${ }^{3}$, \\ H. Levato ${ }^{4}$, M. Grosso ${ }^{4}$, E. Pollmann ${ }^{5}$, C. Buil ${ }^{6}$, and D. C. Knauth ${ }^{7}$ \\ ${ }^{1}$ Ritter Observatory, Dept. of Physics \& Astronomy, The University of Toledo, Toledo, OH 43606-3390, USA \\ 2 Pulkovo Observatory, Saint-Petersburg 196140, Russia \\ ${ }^{3}$ CFHT Corporation, 65-1238 Mamalahoa Hwy, Kamuela, HI 96743, USA \\ ${ }^{4}$ Complejo Astronómico El Leoncito (CASLEO), Casilla de Correo 467, 5400 San Juan, Argentina \\ 5 Charlottenburgerstrasse 26c, 511377 Leverkusen, Germany \\ 66 place Clémence Isaure, 31320 Castanet-Toloson, France \\ 7 Dept. of Physics and Astronomy, John Hopkins University, 3400 N. Charles Str., Baltimore, MD 21218, USA
}

Received 3 April 2003 / Accepted 20 June 2003

\begin{abstract}
We present the results of a spectroscopic monitoring program of the binary system $\delta$ Scorpii, whose primary became a Be star after the last periastron encounter in the Summer of 2000. The observations cover a period of 2 years (March 2001June 2003) and are a continuation of our previous campaign reported in Miroshnichenko et al. (2001). We found that the emission-line spectrum was gradually strengthening over the whole monitoring period. Several short-term increases of the line emission, possibly due to outbursts of matter ejected from the stellar surface, were detected. The outbursts seem to be responsible for the brightness fadings, seen afterwards. We attempt to explain the overall observed line and continuum behaviour in terms of a circumstellar disk, growing in density and size. The disk is most likely Keplerian with an outer radius of $\sim 10 R_{\star}$ and a mean expansion speed of $\sim 0.4 \mathrm{~km} \mathrm{~s}^{-1}$.
\end{abstract}

Key words. techniques: spectroscopic - stars: circumstellar matter - stars: individual: $\delta$ Scorpii - stars: binaries: spectroscopic - stars: Be stars

\section{Introduction}

$\delta$ Sco is one of the brightest stars in the sky. It is also an eccentric binary system with a period of $\sim 10.6$ years, whose orbit has been traced by speckle interferometric observations for about 20 years (e.g., Hartkopf et al. 1996). Until recently, it did not show any line emission. Coté \& van Kerkwijk (1993) detected a weak double-peaked emission that appeared near periastron in 1990. No line strengthening was reported during the 1990's. More details about studies of $\delta$ Sco can be found in Miroshnichenko et al. (2001, hereafter Paper I).

A gradual brightening of $\delta$ Sco in the visual region was observed in June 2000, near the subsequent periastron (Otero et al. 2001). Follow up spectroscopic observations revealed the appearance of line emission. A study of the $\mathrm{H} \alpha$ line behaviour, reported in Paper I, resulted in a refinement of the binary orbit through analysis of the line bisector radial velocities $\left(V_{\mathrm{r}}\right)$, an explanation of a 2-month long brightness

Send offprint requests to: A. S. Miroshnichenko, e-mail: anatoly@physics.utoledo.edu

* Tables 1-2 are also available in electronic form at the CDS via anonymous ftp to cdsarc.u-strasbg.fr $(130.79 .128 .5)$ or via http://cdsweb.u-strasbg.fr/cgi-bin/qcat?J/A+A/408/305 minimum (dip) centered on periastron, and a suggestion that this outburst would be a long-lasting one. In particular, the orbital eccentricity $e=0.94 \pm 0.01$ and the inclination angle of the orbital rotation axis with respect to the line of sight $i=38^{\circ} \pm 5^{\circ}$ were determined in Paper I.

The emission line profiles seen in the spectrum of $\delta$ Sco are very similar to those of classical Be stars and are most likely formed in a flattened, probably quasi-Keplerian, circumstellar (CS) disk. The structure of such disks is not well understood. Only a very few Be stars have recently developed or lost their disks. Examples include $\pi$ Aqr (Bjorkman et al. 2002), which lost its disk over a period of $\sim 6$ years, and 60 Cyg (Koubský et al. 2000), which showed long-term ( $\sim 10$ years) emission line variations. However, the transition stages (from no disk to full disk and back) have not been well documented. Recently Rivinius et al. (1998) presented a detailed spectroscopic study of the early stages of the disk development around the Be star $\mu$ Cen, claiming that this study was the only one to date. Such observations are very important to investigate the mechanisms of disk formation and evolution.

$\delta$ Sco provides a nice opportunity to study the ongoing disk formation process in detail. It is bright (allowing one to obtain high-resolution spectroscopy with relatively small telescopes 
as well as naked eye brightness estimates) and can be observed from both hemispheres nearly 9 months a year. In this paper we report the results of our spectroscopic observations obtained during a period of almost 2 years, immediately following the timeframe discussed in Paper I.

\section{Observations}

Our new spectroscopic observations of $\delta$ Sco were carried out between March 2001 and June 2003 using a number of instruments at different observatories.

At the Ritter Observatory (Toledo, OH, USA) a 1-m telescope equipped with an échelle spectrograph and a Wright Instruments Ltd. CCD camera was employed. The spectra consisted of 9 non-overlapping $\sim 70 \AA$ orders with a spectral resolving power $R \simeq 26000$ covering the wavelength region 5285-6600 A.

A few spectra were obtained during 2 observing runs at the 2.1-m Otto Struve telescope of the McDonald Observatory (Mt. Locke, Texas, USA) with the Sandiford échelle spectrometer (McCarthy et al. 1993) and $R \sim 60000$. A $1200 \times$ 400 pixel CCD was used. The spectra contain 26 overlapping orders in the regions 5530-6830 $\AA$ in October 2001 and 5430-6730 $\AA$ in September 2002. Two spectra were obtained at the 2.7-m Harlan J. Smith telescope of the McDonald Observatory with a coudé spectrograph (Tull et al. 1995 ) providing $R \sim 140000$. These spectra contained 13 nonoverlapping $\sim 40 \AA$-wide orders in a region from $6018 \AA$ to $8943 \AA$.

At the 3.6-m CFHT (Hawaii, USA), the high-resolution $(R \sim 100000)$ Gecko échelle spectrograph, fiber-fed from the Cassegrain focus (Baudrand \& Vitry 2000), and a $2048 \times$ $4608 \times 13.5 \mu \mathrm{m}^{2}$ thinned back-illuminated EEV chip were used. The fiber was continuously agitated to overcome modal noise (Baudrand \& Walker 2000). Three narrow spectral regions centered at $\mathrm{H} \delta(\Delta \lambda \sim 50 \AA)$, He I $5876 \AA$, and $\mathrm{H} \alpha$ $(\Delta \lambda \sim 100 \AA)$ were observed on 2 occasions. At the Complejo Astronómico El Leoncito (Argentina) we used the 2.1-m telescope with the échelle spectrograph REOSC, mounted at the Cassegrain focus and equipped with a $1 \mathrm{~K} \times 1 \mathrm{~K} \mathrm{CCD-chip.}$ This setup allowed us to achieve a $R \sim 15000$. All these data were reduced with IRAF $^{1}$.

For the low-dispersion spectroscopy two small telescopes were used. A 20-cm Cassegrain-Schmidt telescope at the Observatory of the Cologne-Stargazer's Association equipped with a grating-spectrograph and a $768 \times 512$ pixel CCDchip, which provided a $0.395 \AA$ pixel $^{-1}$ reciprocal dispersion, was employed in Germany. A 20-cm Newton telescope equipped with a Littrow grating spectrograph and a cooled KAF1602 Kodak $1728 \times 1024$ pixel CCD, which provided a $0.38 \AA_{\text { pixel }}^{-1}$ reciprocal dispersion, was employed in France (near Toulouse). Both instruments registered a $\sim 200-500 \AA$ interval around the $\mathrm{H} \alpha$ line.

\footnotetext{
${ }^{1}$ IRAF is distributed by the National Optical Astronomy Observatories, which are operated by the Association of Universities for Research in Astronomy, Inc., under contract with the National Science Foundation.
}

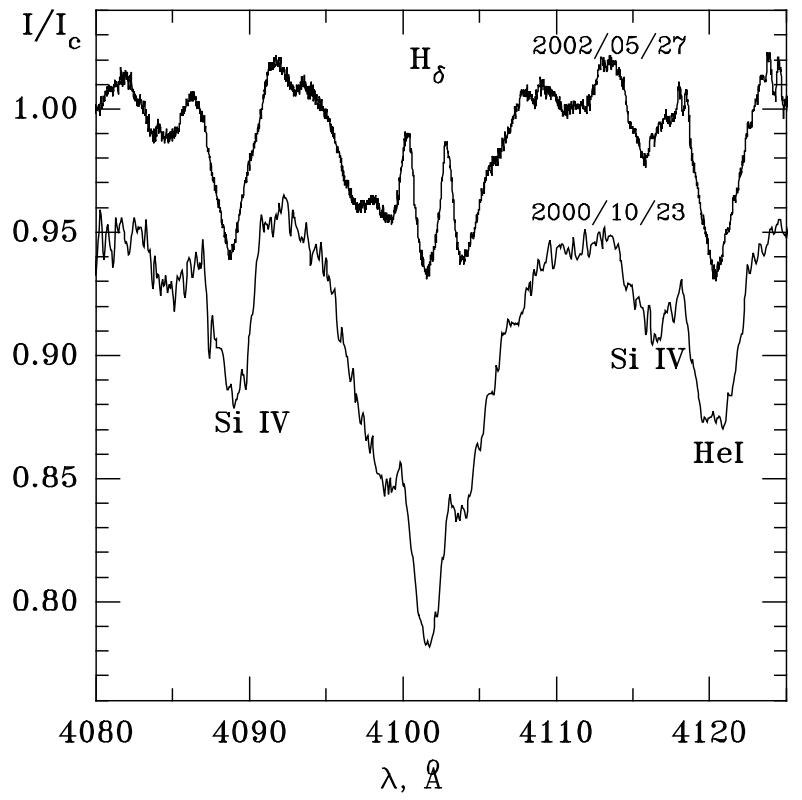

Fig. 1. A portion of the $\delta$ Sco spectrum containing $\mathrm{H} \delta$ and some photospheric lines. The upper spectrum was obtained at CFHT, and the lower one at ESO (described in Paper I).

In total more than 120 high-resolution and 60 lowresolution spectra were obtained. Spectra taken during the same night were averaged. The parameters of the $\mathrm{H} \alpha$ line derived from the high-resolution data are presented in Table 1. This Table contains the same information as in Table 3 of Paper I to allow easy comparison of the two data sets. The $\mathrm{H} \alpha$ equivalent widths $(E W)$ derived from the low-resolution data are shown in Table 2. The mean $V_{\mathrm{r}}$ of the $\mathrm{H} \alpha$ line were measured by matching the symmetric parts of the original and mirrored profiles between intensity levels of $\sim 1.2$ and $1.6-1.8$ in the underlying continuum units. This technique is often used in studies of the $\mathrm{RV}$ variations in Be stars (e.g., Paper I).

\section{Results}

Emission lines were present in the spectrum of $\delta$ Sco over the entire period of our observations. We detected the following emission lines in the observed spectral intervals: $\mathrm{H}$ I lines of the Balmer ( $\mathrm{H} \alpha$ through $\mathrm{H} \delta$ ) and Paschen (P13-P16) series, the He I lines at $\lambda 5876 \AA$, the Fe II lines at $\lambda 5317$ and $6383 \AA$, and the Si II lines at $\lambda 6347$ and $6371 \AA$. At high resolution, they all showed double-peaked profiles typical for quasi-Keplerian disks of Be stars. At low resolution, the peaks of the $\mathrm{H} \alpha$ line were not resolved in some spectra, especially in those obtained in the fall of 2002 and in 2003. The Paschen lines, which we mainly observed in May-June 2002, have double-peaked profiles with peak intensities of 1.1-1.2 of the underlying continuum, peak separations $\Delta V_{\mathrm{r}} \sim 130 \mathrm{~km} \mathrm{~s}^{-1}$, and full widths at half-maximum $F W H M \sim 280 \mathrm{~km} \mathrm{~s}^{-1}$.

We also observed a few absorption lines. From the comparison of our spectra obtained in October 2000 and in May 2002, the absorption lines of Si IV at 4088 and $4116 \AA$ and that of $\mathrm{He}$ I at $4121 \AA$ look virtually the same (Fig. 1). In the yellow region, the $\mathrm{Na}$ I D-lines at $\lambda 5889$ and $5995 \AA$ 
Table 1. Summary of the high-resolution $\mathrm{H} \alpha$ observations of $\delta$ Sco.

\begin{tabular}{|c|c|c|c|c|c|c|c|c|c|c|c|c|c|}
\hline 1 & 2 & 3 & 4 & 5 & 6 & 7 & 1 & 2 & 3 & 4 & 5 & 6 & 7 \\
\hline 1986.922 & $\mathrm{R}$ & 4.85 & $1.68 / 1.69 / 1.57$ & -7.3 & 124 & 290 & 2397.754 & $\mathrm{R}$ & 8.13 & $2.34 / 2.21 / 2.19$ & -3.3 & & 215 \\
\hline 1994.863 & $\mathrm{R}$ & 5.29 & $1.73 / 1.74 / 1.62$ & -6.1 & 117 & 290 & 2398.679 & $\mathrm{R}$ & 8.25 & $2.37 / 2.22 / 2.20$ & -2.1 & 55 & 218 \\
\hline 2003.730 & $\mathrm{R}$ & 5.64 & $1.78 / 1.76 / 1.64$ & -7.8 & 119 & 309 & 2399.741 & $\mathrm{R}$ & 8.39 & $2.39 / 2.25 / 2.24$ & -1.3 & 53 & 217 \\
\hline 2011.439 & $\mathrm{C}$ & 5.24 & $1.79 / 1.75 / 1.63$ & -7.5 & 134 & 280 & 2400.702 & $\mathrm{R}$ & 8.05 & $2.36 / 2.24 / 2.23$ & -1.1 & 50 & 218 \\
\hline 2027.808 & $\mathrm{R}$ & 5.39 & $1.74 / 1.70 / 1.62$ & -5.0 & 138 & 296 & 2404.767 & $\mathrm{R}$ & 8.42 & $2.35 / 2.25 / 2.24$ & -1.3 & 50 & 223 \\
\hline 2028.700 & $\mathrm{R}$ & 5.14 & $1.70 / 1.73 / 1.61$ & -6.8 & 142 & 288 & 2405.747 & $\mathrm{R}$ & 8.49 & $2.38 / 2.28 / 2.27$ & -2.5 & 50: & 218 \\
\hline 2029.783 & $\mathrm{R}$ & 5.45 & $1.70 / 1.71 / 1.63$ & -8.1 & 140 & 299 & 2409.809 & $\mathrm{R}$ & 8.65 & $2.28 / 2.26 / 2.24$ & -3.4 & 50 & 240 \\
\hline 2030.771 & $\mathrm{R}$ & 5.12 & $1.67 / 1.69 / 1.61$ & -5.0 & 130: & 302 & 2413.734 & $\mathrm{R}$ & 8.66 & $2.23 / 2.22 / 2.20$ & -5.3 & 54 & 258 \\
\hline 2033.725 & $\mathrm{R}$ & 5.05 & $1.66 / 1.67 / 1.60$ & -6.6 & & 302 & 2416.728 & $\mathrm{R}$ & 8.81 & $2.24 / 2.27 / 2.24$ & -6.4 & & 261 \\
\hline 2038.718 & $\mathrm{R}$ & 5.04 & $1.70 / 1.69 / 1.59$ & -6.4 & 133 & 288 & 2417.627 & $\mathrm{~L}$ & 8.65 & $2.18 / 2.23 / \ldots$ & -5.0 & $\ldots$ & 259 \\
\hline 2039.796 & $\mathrm{R}$ & 4.89 & $1.67 / 1.65 / 1.59$ & -6.9 & 130 & 295 & 2417.686 & $\mathrm{R}$ & 8.83 & $2.22 / 2.23 /$. & -6.6 & & 272 \\
\hline 2052.705 & $\mathrm{R}$ & 4.90 & $1.72 / 1.67 / 1.60$ & -6.1 & 125 & 284 & 2418.645 & $\mathrm{~L}$ & 8.79 & $2.17 / 2.21 / \ldots$ & -5.5 & & 266 \\
\hline 2059.722 & $\mathrm{R}$ & 5.17 & $1.76 / 1.65 / 1.62$ & -6.3 & 120 & 277 & 2421.763 & $\mathrm{R}$ & 8.77 & $2.18 / 2.20 / 2.17$ & -7.1 & 60: & 280 \\
\hline 2069.712 & $\mathrm{R}$ & 4.91 & $1.76 / 1.67 / 1.63$ & -9.7 & 115 & 277 & 2422.780 & $\mathrm{C}$ & 8.52 & $2.16 / 2.25 / 2.16$ & -6.8 & 65: & 266 \\
\hline 2070.710 & $\mathrm{R}$ & 4.85 & $1.74 / 1.63 / 1.60$ & -8.0 & 115 & 281 & 2426.693 & $\mathrm{R}$ & 8.80 & $2.14 / 2.15 / 2.12$ & -6.7 & 76 & 290 \\
\hline 2074.721 & $\mathrm{R}$ & 4.63 & $1.76 / 1.65 / 1.59$ & -7.3 & 120 & 280 & 2427.736 & $\mathrm{R}$ & 8.45 & $2.12 / 2.13 / 2.08$ & -5.7 & 80 & 291 \\
\hline 2086.664 & $\mathrm{R}$ & 5.10 & $1.76 / 1.68 / 1.61$ & -6.5 & 120 & 283 & 2435.675 & $\mathrm{R}$ & 8.63 & $2.21 / 2.10 / 2.06$ & -7.0 & 98 & 284 \\
\hline 2090.692 & $\mathrm{R}$ & 4.64 & $1.77 / 1.66 / 1.58$ & -9.6 & 120 & 282 & 2441.710 & $\mathrm{R}$ & 9.10 & $2.25 / 2.11 / 2.09$ & -6.7 & 100 & 278 \\
\hline 2092.631 & $\mathrm{R}$ & 5.40 & $1.77 / 1.72 / 1.63$ & -8.0 & 120 & 280 & 2442.634 & $\mathrm{R}$ & 8.98 & $2.25 / 2.09 / 2.09$ & -5.5 & & 276 \\
\hline 2095.600 & $\mathrm{R}$ & 5.21 & $1.78 / 1.69 / 1.63$ & -7.5 & 115 & 281 & 2443.661 & $\mathrm{R}$ & 8.87 & $2.24 / 2.09 / 2.09$ & -6.3 & 105 & 277 \\
\hline 2096.671 & $\mathrm{R}$ & 5.59 & $1.80 / 1.74 / 1.64$ & -6.6 & 117 & 280 & 2444.708 & $\mathrm{R}$ & 8.88 & $2.26 / 2.08 / 2.08$ & -6.9 & 100 & 274 \\
\hline 2100.625 & $\mathrm{R}$ & 4.97 & $1.78 / 1.70 / 1.60$ & -6.2 & 115 & 279 & 2445.619 & $\mathrm{R}$ & 9.05 & $2.27 / 2.11 / 2.10$ & -7.0 & 100 & 275 \\
\hline 2102.620 & M & 4.18 & $1.71 / 1.70 / 1.56$ & -7.0 & 112 & 267 & 2448.622 & $\mathrm{~L}$ & 8.96 & $2.21 / 2.15 / 2.11$ & -3.0 : & 101 & 276 \\
\hline 2103.616 & $\mathrm{R}$ & 5.51 & $1.80 / 1.73 / 1.64$ & -8.0 & 112 & 281 & 2449.707 & $\mathrm{~L}$ & 8.91 & $2.22 / 2.13 / 2.10$ & -3.0 & 100 & 272 \\
\hline 2105.620 & $\mathrm{R}$ & 5.27 & $1.80 / 1.73 / 1.65$ & -7.2 & 112 & 279 & 2450.599 & $\mathrm{~L}$ & 9.14 & $2.15 / 2.05 / 2.04$ & -4.5 & 97 & 270 \\
\hline 2115.625 & $\mathrm{R}$ & 5.39 & $1.81 / 1.76 / 1.66$ & -6.4 & 112 & 281 & 2450.651 & $\mathrm{R}$ & 9.07 & $2.29 / 2.12 / \ldots$ & -6.0 & 105 & 269 \\
\hline 2117.599 & $\mathrm{R}$ & 5.79 & $1.82 / 1.79 / 1.67$ & -7.2 & 112 & 283 & 2454.679 & $\mathrm{R}$ & 9.28 & $2.29 / 2.17 / \ldots$ & -5.5 & 105 & 274 \\
\hline 2121.584 & $\mathrm{R}$ & 5.88 & $1.87 / 1.78 / 1.66$ & -5.7 & 112 & 276 & 2455.663 & $\mathrm{R}$ & 9.25 & $2.28 / 2.18 / \ldots$ & -5.6 & 105: & 275 \\
\hline 2156.529 & $\mathrm{R}$ & 6.79 & $1.94 / 1.88 / 1.77$ & & 100 & 272 & 2455.601 & $\mathrm{~L}$ & 8.93 & $2.22 / 2.16 / \ldots$ & & & 279 \\
\hline 2158.527 & $\mathrm{R}$ & 6.85 & $1.93 / 1.86 / 1.79$ & -5.0 & 100 & 268 & 2456.623 & $\mathrm{R}$ & 9.05 & $2.26 / 2.16 /$ & -5.5 & 100 & 278 \\
\hline 2159.536 & $\mathrm{R}$ & 6.76 & $1.93 / 1.86 / 1.77$ & -8.0 & 100 & 271 & 2456.551 & $\mathrm{~L}$ & 9.06 & $2.25 / 2.21 / 2.20$ & & & 278 \\
\hline 2163.520 & $\mathrm{R}$ & 6.10 & $1.86 / 1.82 / 1.75$ & -6.5 & 100 & 262 & 2457.668 & $\mathrm{R}$ & 9.21 & $2.27 / 2.15 / \ldots$ & -6.4 & 85 & 278 \\
\hline 2174.527 & $\mathrm{R}$ & 5.29 & $1.81 / 1.77 / 1.67$ & 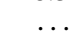 & & 277 & 2458.622 & $\mathrm{R}$ & 9.42 & $2.28 / 2.19 / \ldots$ & -5.2 & 82 & 276 \\
\hline 2175.521 & $\mathrm{R}$ & 5.42 & $1.81 / 1.79 / 1.68$ & & 100 & 279 & 2459.673 & $\mathrm{R}$ & 9.10 & $2.26 / 2.16 / \ldots$ & & 83 & 283 \\
\hline 2182.499 & $\mathrm{R}$ & 5.97 & $1.86 / 1.86 / 1.75$ & -5.0 & 100 & 285 & 2460.651 & $\mathrm{R}$ & 9.45 & $2.25 / 2.21 / \ldots$ & -5.3 & 87 & 283 \\
\hline 2184.487 & $\mathrm{R}$ & 5.82 & $1.82 / 1.82 / 1.74$ & $\ldots$ & 95 & 288 & 2461.641 & $\mathrm{R}$ & 9.78 & $2.28 / 2.23 / 2.22$ & -6.5 & 80 & 279 \\
\hline 2185.500 & $\mathrm{R}$ & 5.54 & $1.83 / 1.79 / 1.69$ & & 100 & 288 & 2462.614 & $\mathrm{R}$ & 9.63 & $2.28 / 2.22 / 2.21$ & -6.3 & 87 & 281 \\
\hline 2186.503 & $\mathrm{R}$ & 5.79 & $1.81 / 1.79 / 1.70$ & & - & 291 & 2463.638 & $\mathrm{R}$ & 9.65 & $2.27 / 2.22 / 2.21$ & -6.2 & 83 & 283 \\
\hline 2187.490 & $\mathrm{R}$ & 5.66 & $1.83 / 1.81 / 1.72$ & $\cdots$ & 95 & 291 & 2541.595 & $\mathrm{M}$ & 9.71 & $2.54 / 2.41 / \ldots$ & -5.0 & & 265 \\
\hline 2192.540 & M & 5.28 & $1.83 / 1.80 / 1.72$ & & 105 & 279 & 2544.559 & M & 9.72 & $2.56 / 2.43 / 2.38$ & & & 259 \\
\hline 2193.541 & M & 6.12 & $1.82 / 1.98 / 1.76$ & $\ldots$ & 102 & 273 & 2684.939 & $\mathrm{R}$ & 10.82 & $2.59 / 2.58 / 2.44$ & -7.0 & 90 & 266 \\
\hline 2194.595 & M & 5.76 & $1.81 / 1.83 / 1.72$ & & 104 & 280 & 2690.885 & $\mathrm{R}$ & 10.25 & $2.49 / 2.49 / 2.36$ & -7.0 & 90 & 268 \\
\hline 2299.930 & $\mathrm{R}$ & 7.15 & $1.97 / 1.95 / 1.89$ & -5.8 & 102 & 274 & 2691.937 & $\mathrm{R}$ & 10.85 & $2.57 / 2.55 / 2.40$ & 1.0 & 87 & 268 \\
\hline 2300.953 & $\mathrm{R}$ & 7.14 & $1.95 / 1.96 / 1.89$ & -5.7 & 100 & 276 & 2697.896 & $\mathrm{R}$ & 10.47 & $2.56 / 2.54 / 2.42$ & $\ldots$ & 85 & 265 \\
\hline 2308.906 & $\mathrm{R}$ & 7.26 & $1.96 / 1.98 / 1.90$ & -5.4 & 95 & 274 & 2705.853 & $\mathrm{R}$ & 10.96 & $2.61 / 2.55 / 2.47$ & & 82 & 260 \\
\hline 2310.917 & $\mathrm{R}$ & 7.26 & $1.97 / 1.98 / 1.90$ & -5.2 & 102 & 277 & 2712.843 & $\mathrm{R}$ & 10.85 & $2.56 / 2.56 / 2.48$ & $\ldots$ & 80 & 268 \\
\hline 2311.904 & $\mathrm{R}$ & 7.31 & $1.97 / 1.98 / 1.90$ & -5.6 & 100 & 277 & 2724.937 & $\mathrm{R}$ & 11.43 & $2.71 / 2.60 / 2.57$ & & 78 & 257 \\
\hline 2314.896 & $\mathrm{R}$ & 7.33 & $1.98 / 2.00 / 1.91$ & -5.4 & 100 & 273 & 2731.849 & $\mathrm{R}$ & 11.09 & $2.78 / 2.67 / 2.54$ & $\ldots$ & 74 & 242 \\
\hline 2319.918 & $\mathrm{R}$ & 7.50 & $1.99 / 2.00 / 1.92$ & -4.9 & 97 & 278 & 2756.772 & $\mathrm{R}$ & 11.32 & $2.90 / 2.76 / \ldots$ & & 37 & 227 \\
\hline 2323.912 & $\mathrm{R}$ & 7.44 & $1.98 / 2.00 / 1.90$ & -4.3 & 99 & 280 & 2757.779 & $\mathrm{R}$ & 11.48 & $2.89 / 2.77 / 2.73$ & & 44 & 227 \\
\hline 2344.830 & $\mathrm{R}$ & 6.79 & $1.90 / 1.93 / 1.84$ & -6.4 & & 284 & 2763.800 & $\mathrm{R}$ & 11.41 & $2.83 / 2.72 / \ldots$ & & 50: & 239 \\
\hline 2356.867 & $\mathrm{R}$ & 7.17 & $1.92 / 1.94 / 1.87$ & -5.8 & 105 & 291 & 2773.692 & $\mathrm{R}$ & 11.57 & $2.73 / 2.72 / 2.67$ & & 50 & 266 \\
\hline 2357.802 & $\mathrm{R}$ & 7.10 & $1.90 / 1.93 / 1.86$ & -7.3 & & 290 & 2780.733 & $\mathrm{R}$ & 11.73 & $2.77 / 2.74 / 2.64$ & $\ldots$ & 63 & 266 \\
\hline 2364.801 & $\mathrm{R}$ & 7.31 & $1.95 / 1.93 / 1.88$ & -7.4 & 103 & 293 & 2781.721 & $\mathrm{R}$ & 11.96 & $2.77 / 2.72 / 2.64$ & & 65 & 264 \\
\hline 2379.719 & $\mathrm{~L}$ & 7.87 & $2.04 / 2.00 / 1.96$ & -5.5 & 99 & 276 & 2782.694 & $\mathrm{R}$ & 11.86 & $2.75 / 2.70 / 2.64$ & $\ldots$ & 68 & 266 \\
\hline 2388.816 & $\mathrm{R}$ & 7.59 & $2.17 / 2.07 / 2.06$ & -3.7 & 75 & 242 & 2791.681 & $\mathrm{R}$ & 11.94 & $2.86 / 2.75 / 2.70$ & & 70 & 248 \\
\hline 2390.738 & $\mathrm{R}$ & 7.92 & $2.21 / 2.13 / 2.10$ & -4.4 & 65 & 238 & 2792.681 & $\mathrm{R}$ & 11.93 & $2.87 / 2.74 / 2.65$ & $\cdots$ & 70: & 248 \\
\hline 2391.749 & $\mathrm{R}$ & 8.02 & $2.25 / 2.14 / 2.12$ & -3.2 & 69 & 229 & & & & & & & \\
\hline
\end{tabular}

The start time of the observation in Modified Heliocentric Julian Dates (MHJD = HJD-2 450 000) is listed in Col. 1; the site where the spectrum was obtained is abbreviated in Col. 2 (R - Ritter Obs., L - Leoncito, C - CFHT, M - McDonald); the line equivalent width in $\AA$ is listed in Col. 3; the intensities of the blue and red emission peaks and of the central depression normalized to the underlying continuum are listed in Col. 4; the mean $V_{\mathrm{r}}$ of the line wings in $\mathrm{km} \mathrm{s}^{-1}$ is given in Col. 5; the peak separation and full width at half-maximum in $\mathrm{km} \mathrm{s}^{-1}$ are given in Cols. 6 and 7, respectively.

Uncertain values are marked with a colon, while the unmeasurable parameters are marked with dots.

exhibited no noticeable variations in either their strengths or $V_{\mathrm{r}}$. Therefore, they have purely an interstellar origin. Weak diffuse interstellar bands at $\lambda$ 5780, 5797, and $6613 \AA$ are seen in our McDonald and Leoncito spectra, in agreement with the presence of weak interstellar extinction in the object's line of sight $\left(A_{V}=0.14 \mathrm{mag}\right.$, Paper I).

The frequently observed emission lines (such as $\mathrm{H} \alpha$, Fe II $\lambda 5317 \AA$, and He I $\lambda 5876 \AA$ ) varied differently. As seen 
Table 2. Summary of the low-resolution $\mathrm{H} \alpha$ observations of $\delta$ Sco.

\begin{tabular}{rrr|rrr|rrr}
\hline \hline MJD & O & $E W$ & MJD & O & $E W$ & MJD & O & $E W$ \\
\hline 2040 & G & 4.8 & 2371 & G & 7.3 & 2519 & G & 12.7 \\
2047 & G & 4.5 & 2387 & G & 8.0 & 2520 & G & 11.3 \\
2048 & F & 5.5 & 2389 & F & 7.7 & 2531 & G & 11.9 \\
2050 & G & 5.4 & 2420 & F & 8.8 & 2679 & F & 12.1 \\
2052 & G & 4.8 & 2424 & G & 8.3 & 2687 & G & 10.6 \\
2055 & G & 5.0 & 2426 & G & 8.7 & 2688 & G & 10.8 \\
2064 & F & 5.4 & 2427 & F & 8.6 & 2690 & G & 10.6 \\
2072 & F & 5.3 & 2442 & F & 9.1 & 2691 & G & 10.7 \\
2075 & F & 5.3 & 2443 & G & 9.1 & 2693 & G & 10.2 \\
2079 & F & 5.4 & 2447 & F & 9.1 & 2695 & G & 9.9 \\
2082 & F & 4.9 & 2450 & G & 8.8 & 2712 & G & 11.6 \\
2084 & F & 5.1 & 2451 & G & 9.9 & 2720 & G & 11.5 \\
2085 & F & 5.3 & 2451 & F & 9.6 & 2724 & G & 11.8 \\
2089 & F & 5.3 & 2463 & F & 10.0 & 2733 & G & 11.6 \\
2090 & F & 5.4 & 2464 & G & 9.1 & 2744 & G & 12.0 \\
2121 & F & 6.1 & 2467 & G & 9.9 & 2747 & F & 11.5 \\
2123 & G & 6.1 & 2473 & G & 10.1 & 2747 & G & 11.6 \\
2127 & F & 6.2 & 2484 & G & 10.8 & 2750 & G & 11.3 \\
2134 & F & 6.6 & 2485 & G & 10.9 & 2753 & G & 11.5 \\
2316 & F & 7.3 & 2503 & G & 10.9 & 2763 & G & 11.8 \\
2358 & F & 7.2 & 2504 & G & 11.0 & 2782 & F & 12.0 \\
2370 & G & 7.3 & 2506 & F & 11.3 & 2788 & G & 12.3 \\
\hline
\end{tabular}

The observation Modified Julian Date (MJD) is listed in Col. 1, the observing site is abbreviated in Col. 2 (F - France, $\mathrm{G}$ - Germany), the line equivalent width in $\AA$ is listed in Col. 3.

in Fig. 1, the $\mathrm{H} \delta$ line became stronger between 2000 and 2002. The $\mathrm{H} \alpha$ line qualitatively showed the same behaviour. The $\mathrm{H} \alpha$ line $E W$ has been growing steadily since the short-term decline just after periastron (see Paper I). The overall $E W$ increase was accompanied by quasi-regular short-term variations (Fig. 2a). The Fe II and He I lines displayed a very modest $E W$ increase with short-term variations of the same order, nearly synchronous with those of the $\mathrm{H} \alpha$ (Fig. 2bc). Their profiles remain similar to those observed in February 2001 and shown in Figs. 2b-d of Paper I.

The line profile characteristics, such as $F W H M$ and $\Delta V_{\mathrm{r}}$, tended to decrease smoothly with time except for a 2-month long sharp minimum in mid-2002 (Fig. 3d), which was accompanied by a shift of the $\mathrm{H} \alpha$ mean $V_{\mathrm{r}}$ (Fig. 3c). A weaker minimum in $F W H M$ and $\Delta V_{\mathrm{r}}$ of $\mathrm{H} \alpha$ was also observed in April 2003. The wings of $\mathrm{H} \alpha$ were gradually widening all the time, showing a full width at the continuum level (FWZI) of $\sim 600 \mathrm{~km} \mathrm{~s}^{-1}$ in October 2000 and $\sim 1200 \mathrm{~km} \mathrm{~s}^{-1}$ in February 2003. At the same time, the wings of the He I $\lambda 5876 \AA$ and the Fe II $\lambda 5317 \AA$ lines remained the same at a FWZI of $\sim 350 \mathrm{~km} \mathrm{~s}^{-1}$. This seems to be due to a smaller optical depth of the latter lines in comparison with $\mathrm{H} \alpha$. The $\mathrm{H} \alpha$ peak intensity ratio $(V / R)$ varied mostly between 0.95 and 1.1 without obvious signs of cyclicity. A slight rise of $V / R$ during the Summer of 2001 and 2002 seen in Table 1 is due to an

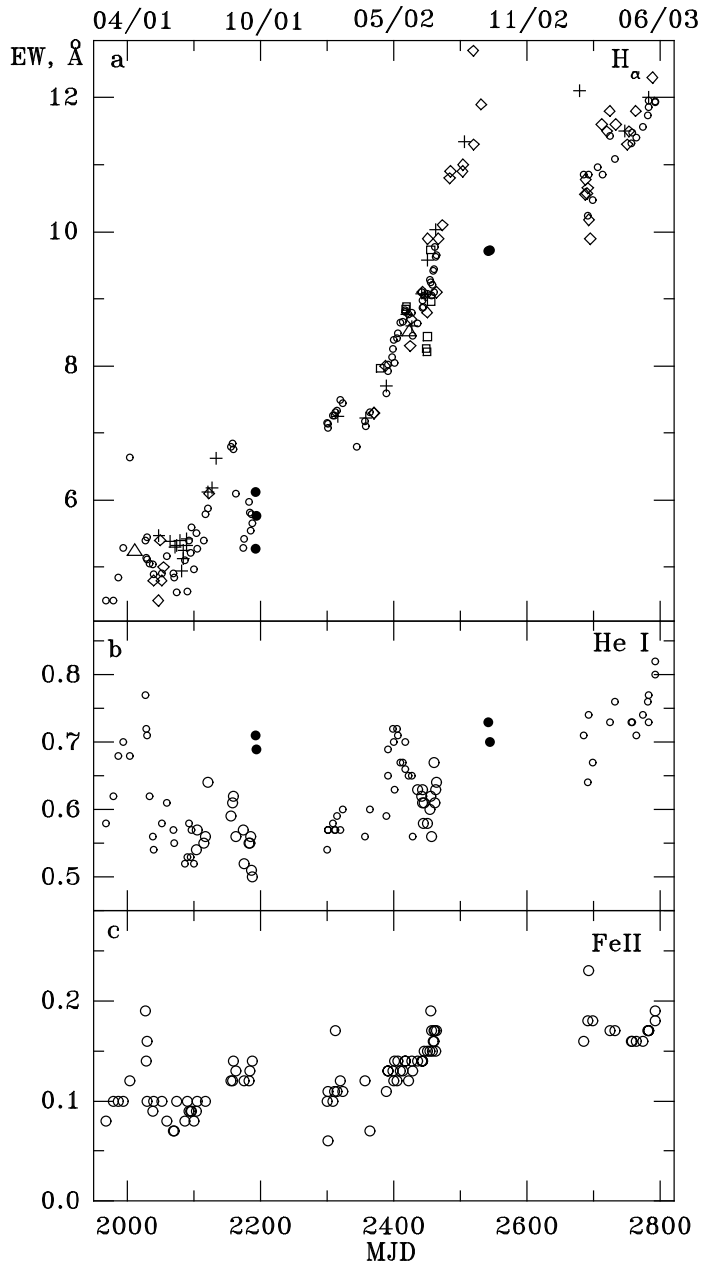

Fig. 2. The equivalent width behaviour of some emission lines in the spectrum of $\delta$ Sco $(\mathrm{H} \alpha, \mathrm{He}$ I $\lambda 5876 \AA$, and Fe II $\lambda 5317 \AA$ from top bottom, respectively). The observations obtained at different sites are denoted by the following symbols: Ritter - open circles, McDonald - filled circles, CFHT - open triangles, Leoncito - open squares, Germany - diamonds, and France - pluses. Some of the He I line profiles are severely affected by telluric lines which increases the uncertainty of the $E W$ measurements. Such data are shown by larger symbols. Calendar dates (MM/YY) are shown at the top.

underestimation of the red peak strength due to a severe contamination by telluric lines.

The mean uncertainties of the $E W$ measurements are as follows: $\sim 0.1 \AA$ for the high-resolution data, $\sim 0.3 \AA$ for the low-resolution data, and $0.03 \AA$ for the He I and Fe II data. These estimates were derived using the analysis by Chalabaev \& Maillard (1983), where a detailed discussion is presented.

In the next section we will analyse the observed phenomena in an attempt to explain their mechanism.

\section{Discussion}

The Balmer line strengthening in the spectrum of $\delta$ Sco indicates that the amount of the CS matter around the primary component is increasing. The double-peaked emission-line profile shape is similar to those of other Be stars, suggesting that the CS matter has a disk-like distribution. In Paper I we used this 


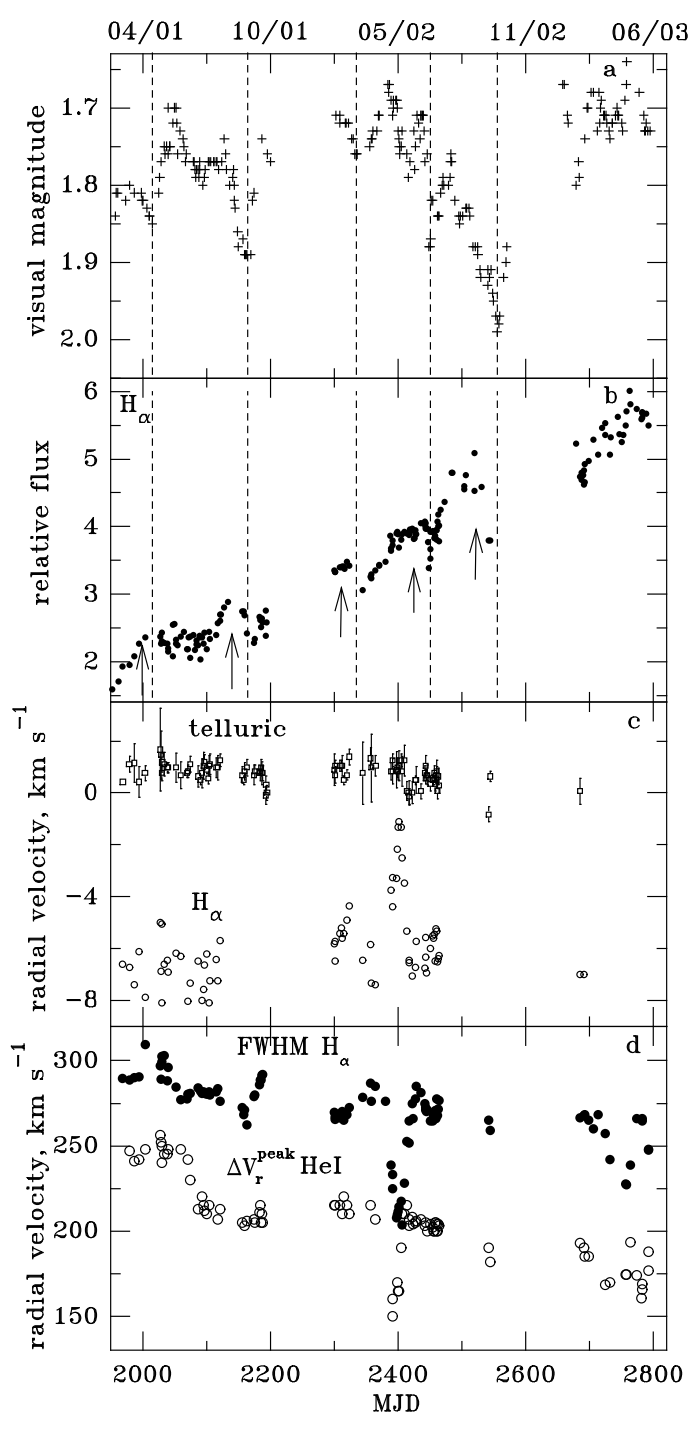

Fig. 3. The brightness and line profiles characteristics of $\delta$ Sco. a) The optical brightness (pluses) variations of $\delta$ Sco from Gandet et al. (2002) and the Kyoto Association of the Variable Stars Observers digest at http://vsnet.kysastro.kyoto-u.ac.jp/ vsnet/gcvs/SCOdelta.html. b) The behaviour of the relative $\mathrm{H} \alpha$ flux calculated with respect to the pre-periastron epoch in July 2000. The arrowhead lines show the positions of short-term $\mathrm{H} \alpha$ flux increases, while the vertical dashed lines show the positions of the brightness decreases. c) The mean $\mathrm{H} \alpha V_{\mathrm{r}}$ (open circles) and the mean $V_{\mathrm{r}}$ of the 6 strongest telluric lines around $\mathrm{H} \alpha$ (open squares) to give a sense of possible systematic errors in the $V_{\mathrm{r}}$ data. d) The behaviour of the $\mathrm{H} \alpha$ line $F W H M$ and the He I line $\Delta V_{\mathrm{r}}$. Calendar dates $(\mathrm{MM} / \mathrm{YY})$ are shown at the top.

assumption to explain a photometric dip that occurred near the periastron time.

The growing $E W$ of $\mathrm{H} \alpha$ along with the decreasing peak separation and $F W H M$ suggest that the disk is expanding (e.g., Zamanov et al. 2001). This is due to a larger contribution of more distant and more slowly rotating matter to the observed profile. The expanding wings of $\mathrm{H} \alpha$, which are mainly due to electron scattering, indicate that the Thomson optical depth and, hence, the mean matter density increases with time. To illustrate this, we use a $\Delta V_{\mathrm{r}}$ versus $E W$ diagramme (Fig. 4).

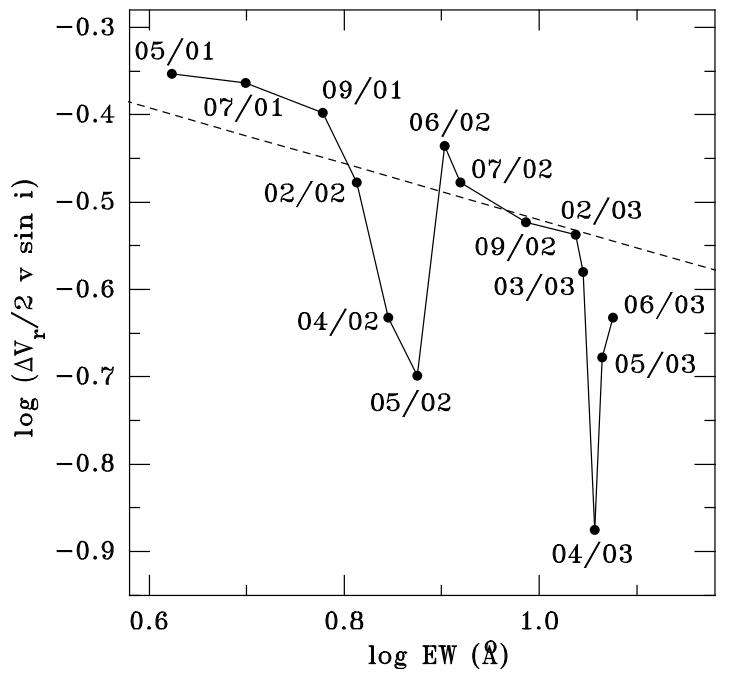

Fig. 4. The peak separation of the $\mathrm{H} \alpha$ line versus its $E W$. The line parameters derived from the profiles obtained within different periods (usually 2-3 weeks long) are averaged. The mean dates in the form $\mathrm{MM} / \mathrm{YY}$ are shown near corresponding data points. The dashed line represents the average relationship between the displayed parameters for Be stars from Zamanov et al. (2001).

A smaller peak separation in combination with a larger $E W$ corresponds to a larger disk size, because of a larger contribution from slower rotating matter to the line emission. At the same time, a larger peak separation at a given $E W$ corresponds to a larger mean matter density. The path of $\delta$ Sco in this plot is very close to the average relationship between $\Delta V_{\mathrm{r}}$ and $E W$ for Be stars. However, in April-May 2002 (MJD 2370-2430) and in April 2003 did $F W H M$ and $\Delta V_{\mathrm{r}}$ drop, while the $E W$ increased slightly. This implies a drop of the mean density in the disk, because the mean density is responsible for the vertical scatter in the diagramme (Zamanov et al. 2001). Temporal evolution of some emission-line profiles is shown in Fig. 5.

More information about the processes in the disk can be extracted from the visual light curve of $\delta$ Sco. It is composed from mostly naked eye estimates, which are however consistent with available photoelectric photometry (Gandet et al. 2002). Assuming that the continuum near $\mathrm{H} \alpha$ varies similarly to the visual light, we calculated the $\mathrm{H} \alpha$ fluxes and normalized them to the pre-periastron level of July 2000, as was done in Paper I. Figure $3 \mathrm{~b}$ shows that the total $\mathrm{H} \alpha$ flux was increasing nearly linearly after periastron. Note that the $\mathrm{H} \alpha$ flux increases near MJD 2000, 2150, 2315, and 2520 were followed by decreases of the visual brightness near MJD 2015, 2165, 2335, and 2555. There is also an indication of a longer-term $\mathrm{H} \alpha$ flux increase around MJD 2390-2420 which was followed by a brightness decrease near MJD 2455. This suggests that the visual brightness variations are most likely caused by the variations in the CS matter, which in turn are due to a variable mass loss rate.

An explanation of this behaviour can be similar to that for the photometric dip, which occurred near periastron, proposed in Paper I. When the mass loss increases, it first affects the spectral lines, which become stronger. The continuum optical depth becomes larger and, after some time as the ejected matter penetrates deeper in the disk (further away from the stellar surface), 

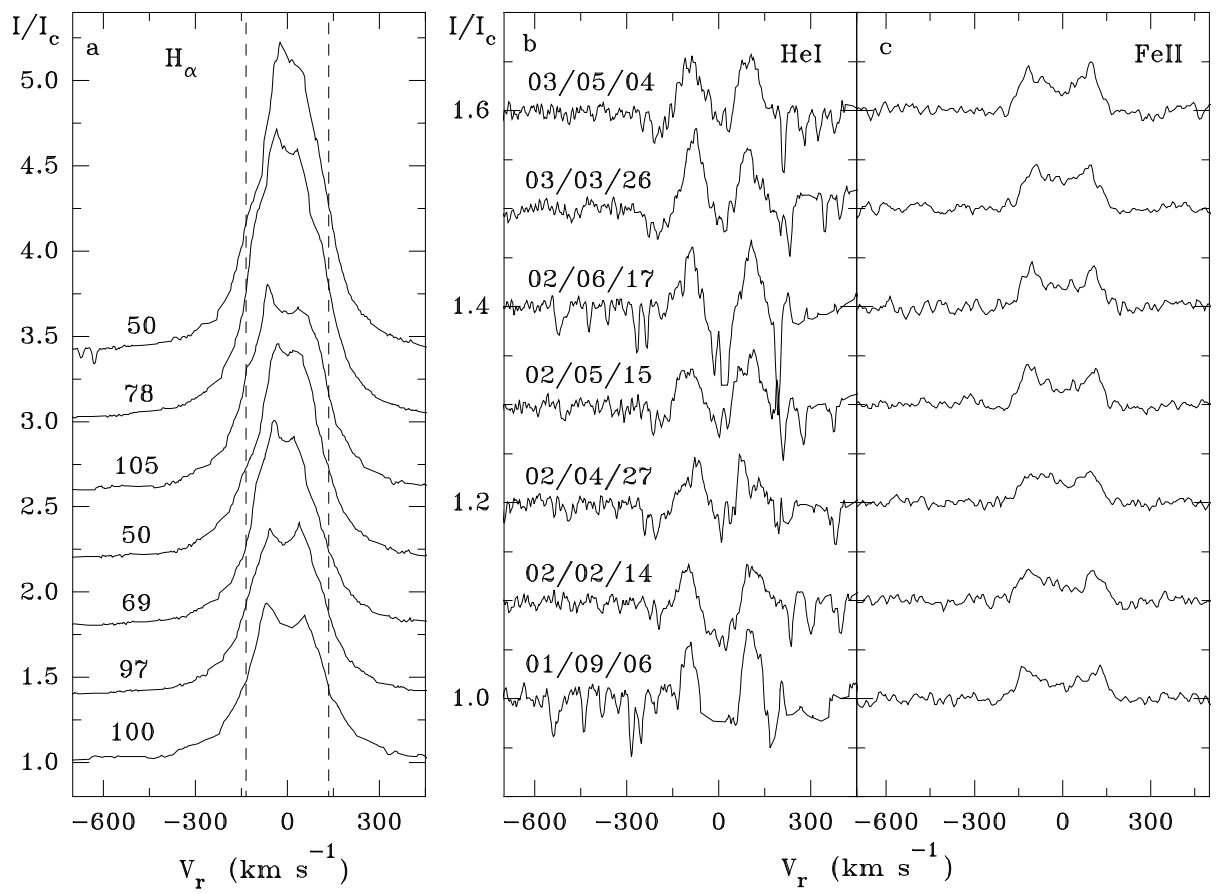

Fig. 5. The emission line profiles of $\delta$ Sco obtained at Ritter Observatory. The H $\alpha$ profiles are shown in panel a), the He I $5876 \AA$ line profiles in panel b), and the Fe II $5317 \AA$ line profiles in panel c). The dashed lines in panel a) represent a $F W H M$ of the H $\alpha$ line immediately before the profile narrowing event in April-May 2002 (see text). The emission peak spearations in $\mathrm{km} \mathrm{s}^{-1}$ are indicated near corresponding H $\alpha$ profiles. Observing dates are indicated in panel b) in the form YY/MM/DD. Telluric lines were not removed from the He I line region. The intensity scale is normalized to the level of the underlying continuum, while the heliocentric RV are given in $\mathrm{km} \mathrm{s}^{-1}$.

the star fades. When the matter is spread out beyond the projection of the CS disk onto the star's disk, the optical depth drops and the star becomes brighter. Subsequent outbursts, occuring close to one another in time, could also produce a cumulative effect on the brightness. Perhaps the deepest brightness minimum near MJD 2555 could be jointly caused by the two outbursts which occurred near MJD 2390-2420 and 2520.

This scenario would also explain the observed FWHM and $\Delta V_{\mathrm{r}}$ minimum. The star does not seem to be rotating at the critical speed. From its projected rotational velocity ( $v_{\text {rot }} \sin i=148 \mathrm{~km} \mathrm{~s}^{-1}$, Brown \& Verschueren 1997) and the inclination of the binary orbit $\left(i=38^{\circ}\right.$, Paper I), which most likely represents the disk orientation, one can calculate that $v_{\text {rot }}=240 \mathrm{~km} \mathrm{~s}^{-1}$. This value is only $\sim 40 \%$ of the critical rotation speed $\left(v_{\text {crit }}=620 \mathrm{~km} \mathrm{~s}^{-1}\right.$ for a primary's mass of $15 M_{\odot}$ and radius of $7 R_{\odot}$ ). At the same time, the innermost parts of the disk need to be rotating at the critical speed (e.g., Porter 1998). Therefore, ejected matter coming on orbit may have an intermediate speed between that of the stellar surface and that of the inner disk boundary. If the amount of this matter is sufficiently large, it would affect the line profiles and account for a temporary decrease of $\Delta V_{\mathrm{r}}$. The $F W H M$ would drop because of the line peak intensity increase. The fact that the line profiles returned to their pre-event shape with a larger $E W$, while the visual brightness remained lower for a longer time, seems to support the above interpretation.

We have observed such a dramatic change in the $\Delta V_{\mathrm{r}}$ and $F W H M$ only on one occasion probably because this was the only spectroscopically well-documented episode coinciding with a major visual fading of the star. At the same time, our data show that a similar phenomenon might have taken place in September 2001 (MJD 2150-2180) and in April 2003 (MJD 2756-2782, see Figs. 3c,d).

The heliocentric RV of the $\mathrm{H} \alpha$ wings (Fig. 3c) remained the same $\left(-7 \pm 1 \mathrm{~km} \mathrm{~s}^{-1}\right)$ most of the time since a strong change during the periastron phase (see Paper I). The only episode of notable change coincided with the $F W H M$ and $\Delta V_{\mathrm{r}}$ drop in April-May 2002 and lasted for about a month. At that time the blue wing became slightly suppressed, so that the whole profile shifted to the red by a few $\mathrm{km} \mathrm{s}^{-1}$. The same effect is observed in the He I $\lambda 5876 \AA$ line. The reason for this phenomenon is not clear. We could speculate that the matter ejection is not confined to the disk plane; if there is some material in the line of sight, the blue wing might be suppressed.

Thus, the overall behaviour of the star's brightness is determined by two major effects. The increasing amount of CS matter in the disk adds additional radiation, while the varying CS optical depth is responsible for the brightness variations. Without the outburst-like matter ejections, the observed brightness would smoothly and asymptotically rise toward $V \sim$ 1.60-1.65 mag. This corresponds to an overall brightening of the star of $\sim 0.7$ mag compared to the quiescent state $(V=$ $2.32 \mathrm{mag}$ ). This is a large brightening that is similar to $\gamma$ Cas (see Hummell 1998), a Be star with a long-lived disk viewed at a similar inclination and a stronger emission-line spectrum. This comparison and an almost constant maximum brightness of $\delta$ Sco in 2001-2002 lead us to suggest that there will be no further significant brightening of the star due to additional CS matter, although the line emission may become stronger. 
The evolution of the emission-line spectrum of $\delta$ Sco can be compared with that of $\mu$ Cen during its CS disk development in 1995-1997 (Rivinius et al. 1998). The smaller sample of our high-resolution spectroscopic observations does not allow us to develop as detailed a picture of line behaviour as was done for $\mu$ Cen. They detected numerous emission line outbursts as the line intensity gradually increased, and separated several stages in the outburst cycle (such as relative quiescence, precursor, outburst, and subsequent relaxation). However, we notice some similarities in the disk growth process for these two stars. The $\mathrm{H} \alpha$ line in the spectra of both stars increased its $E W$ from $\sim 4$ to $\sim 12 \AA$ over a 2 -year period. In both cases several outbursts, which resulted in sudden increases of line emission, were observed. These similarities might imply that the same mechanisms responsible for the matter ejection from the star in $\mu$ Cen (short-period, $\sim 0.5$ day, non-radial pulsations) work in $\delta$ Sco as well. Smith (1986) showed that stable multiple periods are present in the short-term line profile variations of $\delta$ Sco. Also it seems that, like $\mu \mathrm{Cen}, \delta$ Sco is building up a persistent disk (as we suggested in Paper I).

Gandet et al. (2002) noticed cyclical brightness variations with an averaged period of 71 days in the light curve of $\delta$ Sco in 2001-2002. These authors fitted the mean brightness to a parabola. In contrast, the link between the $\mathrm{H} \alpha$ line and brightness behaviour rather suggests that the maximum brightness in 2001-2002 was almost constant, while a number of fadings due to the ejected matter outbursts were observed. The easily recognizable brightness minima occurred at MJD 2015, 2165, 2335, 2450, 2555, and 2680 (see Fig. 3b). The intervals between them are obviously not constant, ranging from 105 to 170 days. It is still unclear if there is any strict periodicity in the brightness variations, since the spectroscopy was less frequent than the brightness estimates and because there may be another cause for the brightness variations.

From our $\mathrm{H} \alpha$ line profile data we can roughly estimate the disk expansion speed. According to Huang (1972), the peak separation is a measure of the outer radius of the emitting disk $\left(\Delta V_{\mathrm{r}} / 2 v \sin i=\left(R_{\text {disk }} / R_{\star}\right)^{-j}\right)$, where $j=0.5$ corresponds to Keplerian rotation and $j=1$ to conservation of angular momentum. Since $\mathrm{H} \alpha$ is formed throughout the ionized part of the disk, the $R_{\text {disk }}$ estimated this way should be close to the real disk edge for such a strong ionizing source as the B0-type $\delta$ Sco primary. Using $2 v \sin i=300 \mathrm{~km} \mathrm{~s}^{-1}, j=0.5, \Delta V_{\mathrm{r}}=130 \mathrm{~km} \mathrm{~s}^{-1}$ in the beginning of 2001, and $\Delta V_{\mathrm{r}}=90 \mathrm{~km} \mathrm{~s}^{-1}$ in the beginning of 2003, one can calculate $R_{\text {disk }}=5.3 R_{\star}$ for the former $\Delta V_{\mathrm{r}}$ and $10.8 R_{\star}$ for the latter. For $j=1 R_{\text {disk }}=2.3 R_{\star}$ and $3.3 R_{\star}$, respectively. Therefore, a mean expansion speed turns out to be $\sim 0.4 \mathrm{~km} \mathrm{~s}^{-1}$ for a Keplerian disk and less than $0.1 \mathrm{~km} \mathrm{~s}^{-1}$ if angular momentum is conserved. The Keplerian assumption seems to be more realistic, because recent calculations show that $R_{\text {disk }}$ of at least $10 R_{\star}$ is needed to explain emission-line strengths and CS continuum excesses similar to those of $\delta$ Sco (e.g., Porter 1999 and Hummell \& Štefl 2001). Nevertheless, even the Keplerian expansion speed is an order of magnitude lower than that suggested by Rivinius et al. (1998) for $\mu$ Cen. On the other hand, it is consistent with the disk dissipation speed for $\pi$ Aqr (Bjorkman et al. 2002). These parameters are still poorly-known due to lack of appropriate observations.
Our data will provide important insight into the processes of disk formation and dissipation.

\section{Conclusions}

Our 2-year (March 2001-June 2003) spectroscopic monitoring of $\delta$ Sco resulted in the following major findings.

1. We observed a steady growth of the CS disk which manifests itself by a continuous strengthening of the emission lines (especially those of the Balmer series) over the whole period of the observations.

2. Short-term emission-line profile variations (intensity increases on top of the gradual intensity rise) seem to be due to outbursts of matter ejection from the stellar surface into the disk. The outbursts are most likely also responsible for the brightness fadings, seen after corresponding line intensity increases.

3. The disk is probably Keplerian with an outer radius of $\sim 10 R_{\star}$, and seems to continue to grow in both density and size.

We encourage observers to follow the $\delta$ Sco disk development using all possible techniques (e.g., spectroscopy, photometry, polarimetry), as it presents a unique opportunity to investigate such a rare event in detail.

Acknowledgements. A.M. and K.S.B. acknowledge support from NASA grant NAG5-8054. Karen Bjorkman is a Cottrell Scholar of the Research Corporation, and gratefully acknowledges their support. Support for observational research at Ritter Observatory has been provided by The University of Toledo, by NSF grant AST-9024802 to B. W. Bopp, and by a grant from the Fund for Astrophysical Research. Technical support is provided by R. J. Burmeister.

\section{References}

Baudrand, J., \& Vitry, R. 2000, in Proc. SPIE, 4008, 182

Baudrand, J., \& Walker, G. A. H. 2001, PASP, 113, 851

Bjorkman, K. S., Miroshnichenko, A. S., McDavid, D. A., \& Pogrosheva, T. M. 2002, ApJ, 573, 812

Brown, A. G. A., \& Verschueren, W. 1997, A\&A, 319, 811

Chalabaev, A., \& Maillard, J. P. 1983, A\&A, 127, 279

Coté, J., \& van Kerkwijk, M. H. 1993, A\&A, 274, 870

Gandet, T. L., Otero, S., Fraser, B., \& West, J. D. 2002, IBVS, 5352

Hartkopf, W. L., Mason, B. D., \& McAlister, H. A. 1996, AJ, 111, 370

Huang, S.-S. 1972, ApJ, 171, 549

Hummell, W. 1998, A\&A, 330, 243

Hummell, W., \& Štefl, S. 2001, A\&A, 368, 471

Koubský, P., Harmanec, P., Hubert, A.-M., et al. 2000, A\&A, 356, 913

McCarthy, J. K., Sandiford, B. A., Boyd, D., \& Booth, J. 1993, PASP, 105,881

Miroshnichenko, A. S., Fabregat, J., Bjorkman, K. S., et al. 2001, A\&A, 377, 485 (Paper I)

Otero, S., Fraiser, B., \& Lloyd, C. 2001, IBVS, 5026

Porter, J. M. 1998, A\&A, 333, L83

Porter, J. M. 1999, A\&A, 348, 512

Rivinius, Th., Baade, D., Štefl, S., Stahl, O., Wolf, B., \& Kaufer, A. 1998, A\&A, 333, 125

Smith, M. A. 1986, ApJ, 304, 728

Tull, R. G., MacQueen, P. J., Sneden, C., \& Lambert, D. L. 1995, PASP, 107, 251

Zamanov, R. K., Reig, P., Martí, J., et al. 2001, A\&A, 367, 884 